\title{
Josephson dynamics of a spin-orbit-coupled Bose-Einstein condensate in a double-well potential
}

\author{
Dan-Wei Zhang, ${ }^{1}$ Li-Bin Fu, ${ }^{2}$ Z. D. Wang, ${ }^{3}$ and Shi-Liang Zhu ${ }^{1,4, *}$ \\ ${ }^{1}$ Laboratory of Quantum Information Technology, School of Physics and Telecommunication Engineering, \\ South China Normal University, Guangzhou, China \\ ${ }^{2}$ Science and Technology Computational Physics Laboratory, Institute of Applied Physics and Computational Mathematics, \\ Beijing 100088, China \\ ${ }^{3}$ Department of Physics and Center of Theoretical and Computational Physics, The University of Hong Kong, \\ Pokfulam Road, Hong Kong, China \\ ${ }^{4}$ Center for Quantum Information, IIIS, Tsinghua University, Beijing, China
}

(Received 17 January 2012; published 11 April 2012)

\begin{abstract}
We investigate the quantum dynamics of an experimentally realized spin-orbit-coupled Bose-Einstein condensate in a double-well potential. The spin-orbit coupling can significantly enhance the atomic interwell tunneling. We find the coexistence of internal and external Josephson effects in the system, which are moreover inherently coupled in a complicated form even in the absence of interatomic interactions. Moreover, we show that the spin-dependent tunneling between two wells can induce a net atomic spin current referred as spin Josephson effects. Such spin Josephson effects can be observable under realistic conditions.
\end{abstract}

DOI: 10.1103/PhysRevA.85.043609

PACS number(s): 03.75.Lm, 67.85.Hj

\section{INTRODUCTION}

Based on the Berry phase effect $[1,2]$ and its non-Abelian generalization [3], the creation of synthetic gauge fields in neutral atoms by controlling atom-light interaction has attracted great interest in recent theoretical studies [4-18] and has been realized in spinor Bose-Einstein condensates (BECs) in the pioneering experiments by Lin et al. [19,20] and also in several subsequent experiments of other groups [21-24]. The neutral atoms in the generated effective Abelian and non-Abelian gauge fields behave like electrons in an electromagnetic field $[19,21,22]$ or electrons with spin-orbit (SO) coupling [20,24]. Different from electrons that are fermions, the atoms with the synthetic SO coupling can be bosons and typically BECs. This bosonic counterpart of the SO coupled materials has no direct analog in solid-state systems and thus has received increasing attention [25-45] for different types of SO coupling, different internal atomic structures (pseudospin- $\frac{1}{2}$, spin-1, and spin-2 bosons, etc.), and different external conditions (homogenous, trapped, and rotated). These theoretical investigations focus mainly on the static properties of SO-coupled BECs and reveal rich phase diagrams of the ground states [26-29,31,32] and exotic vortex structures [34-36,40-42]. However, to our knowledge, their dynamics has been less studied [24,25,33,43-45], wherein the SO-coupled BECs have been demonstrated to exhibit unconventional collective dipole oscillations [24,33] and interesting relativistic dynamics, such as analogs of self-localization [43], Zitterbewegung [44], and Klein tunneling [45] under certain conditions.

On the other hand, quantum dynamics of a BEC in a doublewell potential has been widely investigated. In particular, the coherent atomic tunneling between two wells results in oscillatory exchange of the BEC, which is analogous to the Josephson effects (JEs) for neutral atoms [46-49]. The weakly interacting BECs provide a further context [47-49] for JEs in superconductor systems because they display nonlinear

\footnotetext{
*slzhu@scnu.edu.cn
}

generalizations of typical dc and ac JEs and macroscopic quantum self-trapping (MQST), all of which have been observed in experiments [50-53]. Apart from the conventional single-species BECs [46-49], the Josephson dynamics of two-species BECs [54] and spinor BECs without SO coupling [55] has also been studied [56-60]; however, the dynamics of SO-coupled BECs is yet to be explored.

In this paper, we investigate the dynamics of a specific SO-coupled BEC, which was realized in the experiment by Lin et al. [20], in a double-well trapping potential. We find that the SO coupling in the system increases the atomic tunneling to a large extent, which can significantly enhance the atomic JEs. The full dynamics of the system contains both internal and external JEs, which are moreover inherently coupled in a complicated form even in the absence of interatomic interactions. We further demonstrate that in the small Raman coupling regime the spin-dependent Josephson tunneling can lead to a net atomic spin current by varying conditions, which we refer to as spin Josephson effects. The predicted spin Josephson currents are robust against the parameter adjustment and varying initial conditions and can be observable in a SO-coupled BEC under realistic experimental conditions. We also discuss the Josephson dynamics of the system for the relatively strong and intermediate Raman coupling cases.

The paper is organized as follows. In Sec. II we construct a model that can be used to study the quantum dynamics of a SO-coupled BEC in a double-well potential. Then, in Sec. III, the Josephson dynamics of the constructed system is investigated, with the complicatedly coupled internal and external JEs being addressed. In Sec. IV we demonstrate that in the small Raman coupling regime the spin JEs are exhibited in the system under realistic conditions. In Sec. V, the Josephson dynamics of the system in other Raman coupling regimes is also discussed. A brief discussion and short conclusion are given in Sec. VI.

\section{MODEL}

In a recent experiment, Lin et al. realized a synthetic SO coupling in the ${ }^{87} \mathrm{Rb} \mathrm{BEC}$, in which a pair of Raman 
lasers generated a momentum-sensitive coupling between two internal atomic states [20]. In the bare pseudospin basis $|\uparrow\rangle_{b}=\left|m_{F}=0\right\rangle$ and $|\downarrow\rangle_{b}=\left|m_{F}=-1\right\rangle$, the SO coupling is described by the single-particle Hamiltonian given by [20]

$$
\hat{h}=\frac{\mathbf{p}^{2}}{2 m} \hat{I}+\frac{1}{2}\left(\begin{array}{lc}
\delta & \Omega e^{2 i k_{L} x} \\
\Omega e^{-2 i k_{L} x} & -\delta
\end{array}\right),
$$

where $\mathbf{p}$ is the atomic momentum in the $x y$ plane, $m$ is the atomic mass, $\delta$ is the tunable detuning behaving as a Zeeman field, $k_{L}$ is the wave number of the Raman laser, and $\Omega$ is the Raman coupling strength. Such SO coupling is equivalent to that of an electronic system with equal contribution from Rashba and Dresselhaus SO coupling, and thus it is effective just in one dimension (1D). So we restrict our discussions in $1 \mathrm{D}$ and focus on the motion of atoms along $x$ axis by freezing their $y$ and $z$ degrees of freedom.

To proceed further, we introduce the dressed pseudospins $|\uparrow\rangle=e^{-i k_{L} x}|\uparrow\rangle_{b}$ and $|\downarrow\rangle=e^{i k_{L} x}|\downarrow\rangle_{b}[20,42]$, and then the single-particle Hamiltonian in 1D (along $x$ axis) can be written as

$$
\hat{h}_{0}=\frac{\hbar^{2} \hat{k}_{x}^{2}}{2 m}+2 \alpha \hat{k}_{x} \sigma_{z}+\frac{\Omega}{2} \sigma_{x}+\frac{\delta}{2} \sigma_{z},
$$

where $\hat{k}_{x}$ is the atomic wave vector operator and $\alpha=E_{r} / k_{L}$ is the SO coupling strength with $E_{r}=\hbar^{2} k_{L}^{2} / 2 m$ being the single-photon recoil energy. The dispersion relation of the single-particle Hamiltonian (2) with $\delta=0$ is $E_{ \pm}\left(k_{x}\right)=\frac{\hbar^{2} k_{x}^{2}}{2 m} \pm$ $\sqrt{4 \alpha^{2} k_{x}^{2}+\Omega^{2} / 4}$, which exhibits a structure of two branches. We are interested in the lower energy one, $E_{-}\left(k_{x}\right)$. There is only one minimum in $k_{x}=0$ for large Raman coupling $\Omega>4 E_{r}$, where the atoms of both atomic levels condense. However, the lower branch for $\Omega<4 E_{r}$ presents two minima for condensation of dressed pseudospin-up (left) atoms and dressed pseudo-spin-down (right) atoms, respectively. The Raman coupling and a small $\delta$ modulate the population of atoms in these two states [20]. Here we focus on the later regime (i.e., $\Omega<4 E_{r}$ ) because such BEC with spin-separated and nonzero central momentum is more interesting in contrast to a regular BEC with zero central momentum.

To be more clear, we can rewrite the Hamiltonian (2) as

$$
\hat{h}_{0}=\left(\begin{array}{ll}
H_{\uparrow} & \Omega / 2 \\
\Omega / 2 & H_{\downarrow}
\end{array}\right),
$$

where $\quad H_{\uparrow}=\frac{\hbar^{2}}{2 m}\left(\hat{k}_{x}^{2}+2 k_{L} \hat{k}_{x}\right)+\frac{\delta}{2} \quad$ and $\quad H_{\downarrow}=\frac{\hbar^{2}}{2 m}\left(\hat{k}_{x}^{2}-\right.$ $\left.2 k_{L} \hat{k}_{x}\right)-\frac{\delta}{2}$. Since it is more straightforward to describe the system in terms of dressed pseudospin states instead of bare ones, we work in the dressed pseudospin space and simply refer to dressed pseudospin as spin for convenience hereafter. We also note that the parameters $k_{L}, \Omega$, and $\delta$ in the single-particle Hamiltonian can be tuned independently in a wide range [20], making the SO-coupled BEC a suitable platform for investigating the Josephson dynamics in the presence of SO coupling.

Now we turn to consider such a SO-coupled BEC in a double-well potential denoted by $V(x)$ as shown in Fig. 1(a). Note that the double-well potential here is assumed to be spin independent. To investigate the dynamics of the system, we adopt the two-mode approximation [46-49] with the field

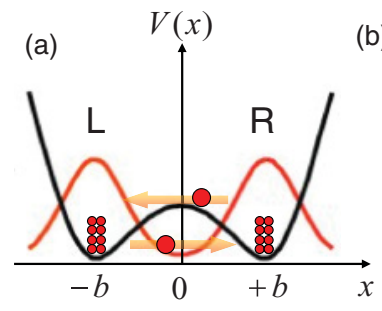

(b)

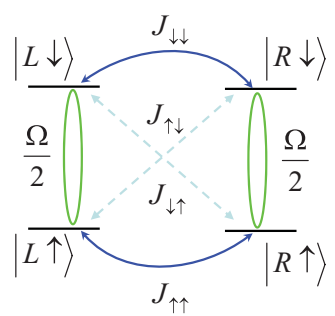

FIG. 1. (Color online) A schematic representation of (a) a SOcoupled BEC in a double-well trap. (b) The dynamic process of the system, where the blue solid and dashed lines represent the interwell tunneling without and with spin flipping, respectively, and the green circles represent the Raman coupling. The interwell spin-flip tunneling induced by the Raman coupling is negligible under current experimental conditions [20,51-53]. The atomic interaction terms are not shown in the figure.

operator

$$
\hat{\Psi}_{\sigma}(x) \simeq \hat{a}_{L \sigma} \psi_{L \sigma}(x)+\hat{a}_{R \sigma} \psi_{R \sigma}(x),
$$

where $\psi_{j \sigma}(x)$ is the ground-state wave function of the $j$ well $(j=L, R)$ with spin $\sigma(\sigma=\uparrow, \downarrow)$ and $\hat{a}_{j \sigma}$ is the annihilation operator for spin $\sigma$ in the $j$ well, satisfying the bosonic commutation relationship $\left[\hat{a}_{j \sigma}, \hat{a}_{k \sigma^{\prime}}^{\dagger}\right]=\delta_{j k} \delta_{\sigma \sigma^{\prime}}$. The validity of the two-mode approximation holds under two conditions: the weak atomic interaction and small effective Zeeman splitting, as the atoms cannot be pumped out of the lowest state of each well under those conditions. In the second quantization formalism, the total Hamiltonian reads

$$
\mathcal{H}=\int d x \hat{\Psi}^{\dagger}(x)\left[\hat{h}_{0}+V(x)+\hat{h}_{\text {int }}\right] \hat{\Psi}(x),
$$

where the two-component field operator $\hat{\Psi}=\left(\hat{\Psi}_{\uparrow}, \hat{\Psi}_{\downarrow}\right)^{\mathrm{T}}$, and the interaction Hamiltonian $\hat{h}_{\text {int }}$ is specified below. By substituting Eq. (4) into Eq. (5), one can rewrite the total Hamiltonian as

$$
\begin{aligned}
\mathcal{H}= & \sum_{j, \sigma} \varepsilon_{j \sigma} \hat{a}_{j \sigma}^{\dagger} \hat{a}_{j \sigma}+\sum_{\sigma \sigma^{\prime}}\left(J_{\sigma \sigma^{\prime}} \hat{a}_{L \sigma}^{\dagger} \hat{a}_{R \sigma^{\prime}}+\text { H.c. }\right) \\
& +\frac{\Omega}{2} \sum_{j}\left(\hat{a}_{j \uparrow}^{\dagger} \hat{a}_{j \downarrow}+\text { H.c. }\right) \\
& +\frac{\delta}{2} \sum_{j}\left(\hat{a}_{j \uparrow}^{\dagger} \hat{a}_{j \uparrow}-\hat{a}_{j \downarrow}^{\dagger} \hat{a}_{j \downarrow}\right)+\mathcal{H}_{\text {int }},
\end{aligned}
$$

where $\varepsilon_{j \sigma}=\int d x \psi_{j \sigma}^{*}(x)\left[H_{\sigma}+V(x)\right] \psi_{j \sigma}(x) \approx \frac{1}{2} \hbar \omega_{j}-E_{r}$ is the single-particle ground-state energy in the $j$ well, $\omega_{j}$ is the harmonic frequency of this well, and $J_{\sigma \sigma}=\int d x \psi_{L \sigma}^{*}(x)\left[H_{\sigma}+V(x)\right] \psi_{R \sigma}(x)$ and $J_{\sigma \bar{\sigma}}=$ $\int d x \psi_{L \sigma}^{*}(x) \frac{\Omega}{2} \psi_{R \bar{\sigma}}(x)$ (with $\sigma$ and $\bar{\sigma}$ referring to different spins) are the tunneling terms schematically shown in Fig. 1(b). In addition, the interaction Hamiltonian is given by

$$
\begin{aligned}
\mathcal{H}_{\text {int }}= & \frac{1}{2} \sum_{j}\left(g_{\uparrow \uparrow}^{(j)} \hat{a}_{j \uparrow}^{\dagger} \hat{a}_{j \uparrow}^{\dagger} \hat{a}_{j \uparrow} \hat{a}_{j \uparrow}+g_{\downarrow \downarrow}^{(j)} \hat{a}_{j \downarrow}^{\dagger} \hat{a}_{j \downarrow}^{\dagger} \hat{a}_{j \downarrow} \hat{a}_{j \downarrow}\right. \\
& \left.+2 g_{\uparrow \downarrow}^{(j)} \hat{a}_{j \uparrow}^{\dagger} \hat{a}_{j \uparrow}^{\dagger} \hat{a}_{j \downarrow} \hat{a}_{j \downarrow}\right),
\end{aligned}
$$

where $g_{\sigma \sigma^{\prime}}^{(j)}=\frac{2 \hbar^{2} a_{\sigma \sigma^{\prime}}}{m l_{\perp}^{2}} \int d x\left|\psi_{j \sigma}(x)\right|^{2}\left|\psi_{j \sigma^{\prime}}(x)\right|^{2}$ is the effective $1 \mathrm{D}$ interacting strength with $a_{\sigma \sigma^{\prime}}$ being the $s$-wave scattering 
length between spin $\sigma$ and $\sigma^{\prime}$ and $l_{\perp}$ being the oscillator length associated to a harmonic vertical confinement. Note that here we have ignored the interwell atomic interactions because the $s$-wave scattering length (which is on the order of nanometers) is much smaller than the interwell distance (which is on the order of micrometers). We have also dropped the interwell coupling since its strength is exponentially smaller than the intrawell counterpart. The Hamiltonian (6) describes the dynamic process of the system schematically shown in Fig. 1(b).

For simplicity, we assume the double-well potential to be symmetric as shown in Fig. 1(a), with each well having the same harmonic trapping frequency $\omega$. Thus we have $\varepsilon_{L}=\varepsilon_{R}$ and $g_{\sigma \sigma^{\prime}}^{(L)}=g_{\sigma \sigma^{\prime}}^{(R)} \equiv g_{\sigma \sigma^{\prime}}$. Such kind of double-well potential can be generated in experiments [52] with the form

$$
V(x)=a\left(x^{2}-b^{2}\right)^{2},
$$

where the parameters $a$ and $b$ are both tunable in the experiments [52]. Expanding $V(x)$ near $x= \pm b$, one obtains its harmonic form as $V^{(2)}(x) \doteq \frac{1}{2} m \omega^{2}(x \pm b)^{2}$ and thus $a=$ $m \omega^{2} / 8 b^{2}$. The ground-state wave functions of the BEC in each well potential with each spin can be approximately represented by its corresponding lowest energy single-particle wave function, which can be worked out by solving the equations $\left[H_{\sigma}+\frac{1}{2} m \omega^{2}(x \pm b)^{2}\right] \psi_{j \sigma}=\varepsilon_{j \sigma} \psi_{j \sigma}$ (here \pm are for $j=L, R$, respectively). The results are [25]

$$
\begin{array}{ll}
\psi_{L \uparrow}=\varphi_{0}^{(L)}(x) e^{-i k_{L} x}, & \psi_{L \downarrow}=\varphi_{0}^{(L)}(x) e^{i k_{L} x}, \\
\psi_{R \uparrow}=\varphi_{0}^{(R)}(x) e^{-i k_{L} x}, & \psi_{R \downarrow}=\varphi_{0}^{(R)}(x) e^{i k_{L} x},
\end{array}
$$

where $\varphi_{0}^{(L)}(x)=\frac{1}{\sqrt{l_{0} \sqrt{\pi}}} e^{-(x+d)^{2} / 2 l_{0}^{2}} \quad$ and $\quad \varphi_{0}^{(R)}(x)=\frac{1}{\sqrt{l_{0} \sqrt{\pi}}}$ $e^{-(x-d)^{2} / 2 l_{0}^{2}}$ with $l_{0}=\sqrt{\hbar / m \omega}$ being the oscillator length. By substituting Eq. (9) into the expressions of $J_{\sigma \sigma^{\prime}}$, one can obtain

$$
\begin{gathered}
J_{\uparrow \uparrow}=J^{(T)}+J^{(\mathrm{SO})}+J^{(V)}+J^{(Z)}, \\
J_{\downarrow \downarrow}=J^{(T)}+J^{(\mathrm{SO})}+J^{(V)}-J^{(Z)}, \\
J_{\uparrow \downarrow}=J_{\downarrow \uparrow}=J^{(R)},
\end{gathered}
$$

where the terms $J^{(T)}=-\frac{\hbar^{2}}{2 m} \int d x \varphi_{0}^{(L)} \varphi_{0}^{\prime \prime(R)}, \quad J^{(\mathrm{SO})}=$ $-E_{r} \int d x \varphi_{0}^{(L)} \varphi_{0}^{(R)}, \quad J^{(V)}=\int d x \varphi_{0}^{(L)} V(x) \varphi_{0}^{(R)}, \quad J^{(Z)}=$ $\frac{\delta}{2} \int d x \varphi_{0}^{(L)} \varphi_{0}^{(R)}$, and $J^{(R)}=\frac{\Omega}{2} \int d x \varphi_{0}^{(L)} e^{-2 i k_{L} x} \varphi_{0}^{(R)}$.

Compared with the atomic tunneling of a regular BEC, the SO-coupled BEC in this system exhibits two additional tunneling channels, the SO-coupling-induced tunneling term $J^{(\mathrm{SO})}$ and the Raman-coupling-induced one $J^{(R)}$. To clarify the effects of these terms in the tunneling processes, we need to work out and compare the energy scales of all the terms $J^{(\eta)}$, where $\eta=\{T, V, Z, \mathrm{SO}, R\}$. Substituting Eqs. (8) and (9) into Eq. (10), we can obtain the following analytical solutions:

$$
J^{(\eta)}=\xi_{\eta} e^{-b^{2} / l_{0}^{2}},
$$

where $\xi_{T}=\frac{\hbar^{2}}{2 m l_{0}^{2}}\left(\frac{1}{2}-\frac{b^{2}}{l_{0}^{2}}\right), \xi_{V}=\frac{\hbar^{2}}{8 m b^{2} l_{0}^{4}}\left(\frac{3}{4} l_{0}^{4}-b^{2} l_{0}^{2}+b^{4}\right), \xi_{Z}=$ $\delta / 2, \xi_{\text {SO }}=-E_{r}$, and $\xi_{R}=\Omega e^{-k_{L}^{2} l_{0}^{2}}$. Since the Zeeman field $\delta$ is independently tunable to the double-well structure and should be small, we here further assume $\delta \ll E_{r}$ and thus we focus on the comparison among $J^{(\mathrm{SO}, R, T, V)}$. The effects of Zeemansplitting-induced tunneling are specified in the Sec. IV.

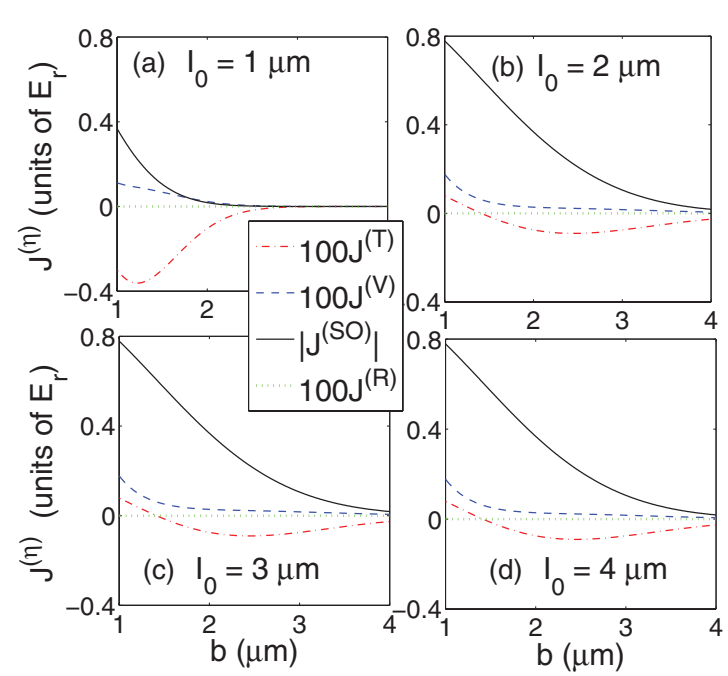

FIG. 2. (Color online) The energy scales of tunneling terms $J^{(\eta)}$ as a function of $b$ for (a) $l_{0}=1 \mu \mathrm{m}$, (b) $l_{0}=2 \mu \mathrm{m}$, (c) $l_{0}=3 \mu \mathrm{m}$, and (d) $l_{0}=4 \mu \mathrm{m}$, respectively. In (a)-(d) we set $\Omega=0.25 E_{r}$ with unit $E_{r}=22.5 \mathrm{kHz}$.

For $l_{0} \sim \sqrt{2} b$, we have $\xi_{T} \sim 0$ and $\xi_{V} \sim \frac{\hbar^{2}}{4 m b^{2}}$, and thus $\frac{\left|\xi_{\text {so }}\right|}{\left|\xi_{V}\right|}=8 \pi^{2}\left(\frac{b}{\lambda_{L}}\right)^{2} \gtrsim 100$. Here we have assumed the same wavelength $\lambda_{L}=2 \pi / k_{L}=0.8 \mu \mathrm{m}$ and recoil frequency $E_{r} / \hbar=22.5 \mathrm{kHz}$ as those in the experiments [20], and $b$ and $l_{0}$ are on the order of micrometers [51-53]. In fact, in the regime of $b^{2} / l_{0}^{2} \sim[0.5,2]$, we find that

$$
\left|\xi_{\text {SO }}\right| \gtrsim 100 \max \left\{\left|\xi_{T}\right|,\left|\xi_{V}\right|\right\} .
$$

Besides, one can check that $\xi_{R} \sim \Omega e^{-64}$ for $l_{0} \sim 1 \mu \mathrm{m}$ and $\lambda_{L}=0.8 \mu \mathrm{m}$, and thus the Raman-coupling-induced tunneling is negligible in this system. The comparisons among $J^{(\eta)}$ for some typical parameters are shown in Fig. 2. In other words, we find that under realistic experimental conditions [20,5153], the spin-flipping tunneling induced by Raman coupling is negligible but the SO-coupling-induced tunneling term $J^{(\mathrm{SO})}$ dominates and moreover it greatly enhances atomic tunneling in this system. Thus we may rewrite the tunneling terms as

$$
J_{\uparrow \downarrow}=J_{\downarrow \uparrow} \approx 0, \quad J_{\uparrow \uparrow} \approx J_{\downarrow \downarrow} \approx J^{(\mathrm{SO})}=-\gamma E_{r},
$$

where $\gamma=\exp \left(-b^{2} / l_{0}^{2}\right) \sim[0.1,0.6]$. It is worthwhile to note that the new tunneling terms $J^{(\mathrm{SO})}$ and $J^{(R)}$ in this SO-coupled system are both tunable, enabling us to study the interesting effects of SO coupling in the atomic interwell tunneling. For instance, one can decrease the effective wave number in $x$ axis to the scale $k_{L} \sim 1 / l_{0}$ so that $J^{(R)} \sim 0.37 \Omega$ and then the Raman-coupling-induced tunneling can revive. This can be achieved by adjusting the angle between the applying Raman lasers and the trapping potential or alternatively by using lasers with larger wavelength. In addition, in the same way one can tune the recoil energy $E_{r}$ to identify the enhancement of atomic tunneling due to the SO coupling (i.e., the effect of $J^{(\mathrm{SO})}$ ) in experiments. As a first step to investigate the system under current experimental conditions, we here concentrate on the tunneling regime governed by Eq. (13).

In the previous experiments of JEs in a rubidium BEC in a double-well trap [51-53], the trapping frequency of individual well $\omega$ is on the order of $0.1 E_{r} / \hbar$ (i.e., several kilohertz). In the 
absence of SO coupling [47-49], the atomic tunneling energy $\left|J^{(T)}+J^{(V)}\right|$ is just typically up to $0.001 E_{r}$ (about $\hbar \times 20$ $\mathrm{Hz}$ ) [53]. The energy scale of Zeeman field $\delta$ in the experiment [20] is tunable and can be as large as $0.01 E_{r}$, which is small compared to $J^{(\mathrm{SO})}$ but is still one order larger than those of $\left|J^{(T, V)}\right|$. Besides, all the terms $J^{(\eta)}$ are tunable in experiments and contribute to atomic tunneling together. Therefore, when investigating the Josephson dynamics of this system under realistic conditions, we do not specify the contribution from $J^{(T, V)}$ in tunneling for both cases of the zero and nonzero (but small) Zeeman field.

\section{FULL DYNAMICS OF THE SYSTEM}

We are now in the position to investigate the quantum dynamics of the system constructed in the previous section. We first address the noninteracting case, that is, $\mathcal{H}_{\text {int }}=0$ in Eq. (6), in which the single-particle Hamiltonian is given by

$$
\begin{aligned}
\mathcal{H}_{0} \simeq & J_{\uparrow \uparrow}\left(\hat{a}_{L \uparrow}^{\dagger} \hat{a}_{R \uparrow}+\hat{a}_{L \uparrow} \hat{a}_{R \uparrow}^{\dagger}\right)+J_{\downarrow \downarrow}\left(\hat{a}_{L \downarrow}^{\dagger} \hat{a}_{R \downarrow}+\hat{a}_{L \downarrow} \hat{a}_{R \downarrow}^{\dagger}\right) \\
& +\frac{\Omega}{2}\left(\hat{a}_{L \uparrow}^{\dagger} \hat{a}_{L \downarrow}+\hat{a}_{L \uparrow} \hat{a}_{L \downarrow}^{\dagger}+\hat{a}_{R \uparrow}^{\dagger} \hat{a}_{R \downarrow}+\hat{a}_{R \uparrow} \hat{a}_{R \downarrow}^{\dagger}\right) \\
& +\frac{\delta}{2}\left(\hat{a}_{L \uparrow}^{\dagger} \hat{a}_{L \uparrow}-\hat{a}_{L \downarrow}^{\dagger} \hat{a}_{L \downarrow}+\hat{a}_{R \uparrow}^{\dagger} \hat{a}_{R \uparrow}-\hat{a}_{R \downarrow}^{\dagger} \hat{a}_{R \downarrow}\right) .
\end{aligned}
$$

Here we have dropped the tunneling terms $J_{\sigma \bar{\sigma}}$ since the spin-flipping tunneling amplitude can be neglected in the current experimental conditions [20,51-53]. In order to study the dynamic properties of the system, we need to work with the equations of motion. The corresponding Heisenberg equations read

$i \hbar \frac{d}{d t} \hat{a}_{j \sigma}=\left[\hat{a}_{j \sigma}, \mathcal{H}_{0}\right]=J_{\sigma \sigma} \hat{a}_{\bar{j} \sigma}+\frac{\Omega}{2} \hat{a}_{j \bar{\sigma}}+(-1)^{p} \frac{\delta}{2} \hat{a}_{j \sigma}$,

where $\sigma$ and $\bar{\sigma}$ refer to different spins, $j$ and $\bar{j}$ refer to different wells, and $p=0,1$ are for $\sigma=\uparrow, \downarrow$, respectively. Using the mean-field approximation, one has $\hat{a}_{j \sigma} \simeq\left\langle\hat{a}_{j \sigma}\right\rangle \equiv a_{j \sigma}$ with $a_{j \sigma}$ being $c$ numbers. Thus we can rewrite the equations of motion as

$$
i \hbar \dot{a}_{j \sigma}=J_{\sigma \sigma} a_{\bar{j} \sigma}+\frac{\Omega}{2} a_{j \bar{\sigma}}+(-1)^{p} \frac{\delta}{2} a_{j \sigma} .
$$

By defining a four-component wave function $\Phi=$ $\left(a_{L \uparrow}, a_{L \downarrow}, a_{R \uparrow}, a_{R \downarrow}\right)^{\mathrm{T}}$, Eq. (16) is rewritten as $i \hbar \frac{d}{d t} \Phi=H_{M} \Phi$, where the Hamiltonian of the system is given by

$$
H_{M}=\left(\begin{array}{cccc}
\frac{\delta}{2} & \frac{\Omega}{2} & J_{\uparrow \uparrow} & 0 \\
\frac{\Omega}{2} & -\frac{\delta}{2} & 0 & J_{\downarrow \downarrow} \\
J_{\uparrow \uparrow} & 0 & \frac{\delta}{2} & \frac{\Omega}{2} \\
0 & J_{\downarrow \downarrow} & \frac{\Omega}{2} & -\frac{\delta}{2}
\end{array}\right) .
$$

We now look into the JEs in this system. Let us further express $a_{j \sigma}$ as $a_{j \sigma}=\sqrt{N_{j \sigma}} e^{i \theta_{j \sigma}}$, where the particle numbers $N_{j \sigma}$ and phases $\theta_{j \sigma}$ are all time-dependent in general. According to Eq. (16), we can obtain

$$
\begin{aligned}
i \hbar \frac{\dot{N}_{j \sigma}}{2}-\hbar N_{j \sigma} \dot{\theta}_{j \sigma}= & J_{\sigma \sigma} \sqrt{N_{j \sigma} N_{\bar{j} \sigma}} e^{i\left(\theta_{\bar{j} \sigma}-\theta_{j \sigma}\right)} \\
& +\frac{\Omega}{2} \sqrt{N_{j \sigma} N_{j \bar{\sigma}}} e^{i\left(\theta_{j \bar{\sigma}}-\theta_{j \sigma}\right)}+(-1)^{p} \frac{\delta}{2} N_{j \sigma} .
\end{aligned}
$$

Separating the imaginary and real parts of Eq. (18) yields two groups of equations as

$$
\begin{aligned}
\dot{N}_{j \sigma}= & \frac{2 J_{\sigma \sigma}}{\hbar} \sqrt{N_{j \sigma} N_{\bar{j} \sigma}} \sin \left(\theta_{\bar{j} \sigma}-\theta_{j \sigma}\right) \\
& +\frac{\Omega}{\hbar} \sqrt{N_{j \sigma} N_{j \bar{\sigma}}} \sin \left(\theta_{j \bar{\sigma}}-\theta_{j \sigma}\right), \\
\dot{\theta}_{j \sigma}= & -\frac{J_{\sigma \sigma}}{\hbar} \sqrt{\frac{N_{\bar{j} \sigma}}{N_{j \sigma}}} \cos \left(\theta_{\bar{j} \sigma}-\theta_{j \sigma}\right) \\
& -\frac{\Omega}{2 \hbar} \sqrt{\frac{N_{j \bar{\sigma}}}{N_{j \sigma}}} \cos \left(\theta_{j \bar{\sigma}}-\theta_{j \sigma}\right)-(-1)^{p} \frac{\delta}{2 \hbar} .
\end{aligned}
$$

Equation (19) actually consists of eight coupled equations. To simplify these equations, we introduce $\phi_{\sigma}=\theta_{R \sigma}-\theta_{L \sigma}$ and $\rho_{\sigma}=N_{R \sigma}-N_{L \sigma}$ for the phase and particle number differences between two wells with the same spin $\sigma$, and $\phi_{j}=\theta_{j \downarrow}-\theta_{j \uparrow}$ and $\rho_{j}=N_{j \downarrow}-N_{j \uparrow}$ for the phase and particle number differences between two spins in the same well $j$, respectively. Thus we can obtain

$$
\begin{aligned}
& \dot{\rho}_{\sigma}=\mathcal{L}_{1} \sin \phi_{\sigma}+\sum_{j}(-1)^{p+q} \mathcal{L}_{2} \sin \phi_{j}, \\
& \dot{\rho}_{j}=\sum_{\sigma}(-1)^{p+q} \mathcal{L}_{1} \sin \phi_{\sigma}+\mathcal{L}_{2} \sin \phi_{j},
\end{aligned}
$$

where $\mathcal{L}_{1}=-\frac{2 J_{\sigma \sigma}}{\hbar} \sqrt{N_{R \sigma} N_{L \sigma}}, \mathcal{L}_{2}=-\frac{\Omega}{\hbar} \sqrt{N_{j \uparrow} N_{j \downarrow}}$, and $q=$ 0,1 for $j=R, L$, respectively ( $p=0,1$ for $\sigma=\uparrow, \downarrow$, respectively). From Eq. (20), we find the coexistence of internal JE related to $\dot{\rho}_{j}\left(\phi_{j}\right)$ and external JE related to $\dot{\rho}_{\sigma}\left(\phi_{\sigma}\right)$. Moreover, the internal and external JEs are inherently coupled in a more complicated form.

Before ending this section, we briefly discuss the weakly interacting case, which has been assumed to meet the requirement of two-mode approximation. In this regime, the mean-field analysis still works well, and the dropped term $\mathcal{H}_{\text {int }}$ can be taken into account within the previous discussions. This leads to two additional terms related to interactions into Eq. (16), and now the equations of motion are given by

$$
\begin{aligned}
i \hbar \dot{a}_{j \sigma}= & J_{\sigma \sigma} a_{\bar{j} \sigma}+\frac{\Omega}{2} a_{j \bar{\sigma}}+(-1)^{p} \frac{\delta}{2} a_{j \sigma} \\
& +g_{\sigma \sigma}\left|a_{j \sigma}\right|^{2} a_{j \sigma}+g_{\sigma \bar{\sigma}}\left|a_{j \bar{\sigma}}\right|^{2} a_{j \sigma},
\end{aligned}
$$

where the interacting strength $g_{\sigma \sigma^{\prime}}$ can be found as $g_{\sigma \sigma^{\prime}}=$ $\frac{\sqrt{2} \hbar^{2} a_{\sigma \sigma^{\prime}}}{\sqrt{\pi} m l_{\perp}^{2} l_{0}}$. The estimation of the interaction energy and the Josephson dynamics in the presence of weak interactions are presented in the next section.

\section{SPIN JOSEPHSON EFFECTS IN WEAK RAMAN COUPLING REGIME}

In the preceding section, we have shown that the SOcoupled BEC in a double-well potential exhibits the complicated coupled external and internal Josephson dynamics. In this section, we consider a specific dynamic process of the system in the weak (but nonvanishing) Raman coupling regime (i.e., $\Omega / E_{r}, \Omega /\left|J_{\sigma \sigma}\right| \ll 1$ ), so that the synthetic SO coupling is still present with strengths almost independent on $\Omega$. Thus the external Josephson dynamics dominates by the tunnelings 
$J_{\sigma \sigma}$. In fact, the manipulation and detection of the SO-coupled BECs in this regime have been performed in experiments [20].

For the weak Raman coupling, we find that the ratio $v \equiv\left|J_{\sigma \sigma}\right| / \Omega$ can reach several hundreds from Eq. (13). Thus within the time scale $\tau \sim \hbar / \Omega \simeq 45 \mathrm{~ms}$ for $\Omega=0.001 E_{r}$, one can ignore the effects of the spin flipping, which leads to two external Josephson tunneling processes for different spins. The spins in this regime are conserved and then the total particle number of spin $\sigma$ denoted by $N_{\sigma t}=N_{L \sigma}+N_{R \sigma}$ are timeindependent constants. We assume $N_{\uparrow t}=N_{\downarrow t}=N_{t} / 2$ for simplicity, where $N_{t}=\sum_{j, \sigma} N_{j \sigma}$ is the total atomic number. The equations of motion (21) in this case can be rewritten as

$$
i \hbar \frac{d}{d t}\left(\begin{array}{c}
a_{L \sigma} \\
a_{R \sigma}
\end{array}\right)=\left(\begin{array}{cc}
(-1)^{p} \frac{\delta}{2}+g_{\sigma \sigma}\left|a_{L \sigma}\right|^{2}+g_{\sigma \bar{\sigma}}\left|a_{L \bar{\sigma}}\right|^{2} & J_{\sigma \sigma} \\
J_{\sigma \sigma} & (-1)^{p} \frac{\delta}{2}+g_{\sigma \sigma}\left|a_{R \sigma}\right|^{2}+g_{\sigma \bar{\sigma}}\left|a_{R \bar{\sigma}}\right|^{2}
\end{array}\right)\left(\begin{array}{c}
a_{L \sigma} \\
a_{R \sigma}
\end{array}\right) .
$$

By defining the normalized interwell particle number difference for spin $\sigma$ as $\mathcal{Z}_{\sigma}=\left[N_{R \sigma}-N_{L \sigma}\right] / N_{\sigma t}$ $\left(-1 \leqslant \mathcal{Z}_{\sigma} \leqslant 1\right)$, the equations of motion (19) become rather simple in this case (similar to those for the regular two species BECs [56]), which are given by

$$
\begin{aligned}
& \dot{\mathcal{Z}}_{\sigma}=-\frac{2 J_{\sigma \sigma}}{\hbar} \sqrt{1-\mathcal{Z}_{\sigma}} \sin \phi_{\sigma}, \\
& \dot{\phi}_{\sigma}=\frac{J_{\sigma \sigma}}{\hbar} \frac{\mathcal{Z}_{\sigma}}{\sqrt{1-\mathcal{Z}_{\sigma}^{2}}} \cos \phi_{\sigma}+\frac{U_{\sigma \sigma}}{\hbar} \mathcal{Z}_{\sigma}+\frac{U_{\sigma \bar{\sigma}}}{\hbar} \mathcal{Z}_{\bar{\sigma}},
\end{aligned}
$$

with the interaction energy $U_{\sigma \sigma^{\prime}}=\frac{N_{t}}{2} g_{\sigma \sigma^{\prime}}$. The spin-dependent atomic density current is given by

$$
I_{\sigma}=\frac{N_{t}}{2} \cdot \dot{\mathcal{Z}}_{\sigma}
$$

From Eq. (24), we can define the net spin current as

$$
I_{s}=I_{\uparrow}-I_{\downarrow}
$$

and the total atomic current as

$$
I_{a}=I_{\uparrow}+I_{\downarrow} .
$$

We first consider the JEs of the system in the noninteracting limit, that is, $U_{\sigma \sigma}=U_{\sigma \bar{\sigma}}=0$ in Eq. (23), which can be realized by Feshbach resonance [61]. Under this condition, the two external Josephson tunneling processes for different spins are decoupled. We numerically calculate Eqs. (23), with some typical results of the time evolution of $\mathcal{Z}_{\sigma}$ for different initial conditions shown in Fig. 3. In the calculations, we have assumed the zero Zeeman field $\delta=0$ in Figs. 3(a) and 3(b) and small Zeeman field $\delta=0.01 E_{r}$ in Figs. 3(c) and 3(d). Compared with zero-Zeeman-field cases, a small Zeeman field results in a deviation in Josephson tunneling strengths $J_{\sigma \sigma}$ for different spins. Here $\mathcal{Z}_{\uparrow}(t)$ and $\mathcal{Z}_{\downarrow}(t)$ demonstrate the oscillatory Josephson tunnelings, which are similar to the early results in Ref. [48]. As shown in Figs. 3(a)-3(d), they are spin dependent, and the dynamic evolution of each one depends on its own tunneling strength, phase, and initial conditions. We also calculate $I_{\sigma}, I_{s}$, and $I_{a}$ in this regime with typical results shown in Fig. 4. It is interesting to see that the spin-dependent atomic density currents due to the spin-related Josephson tunnelings give rise to a net spin current (cf. Fig. 4), and moreover in some certain initial conditions the total atomic current can be zero, which leads to an interesting pure spin current [cf. Fig. 4(b)]. We call such JEs spin Josephson effects, which can be observable in experiments by measuring the time evolution of spin-dependent population imbalance of the atomic gas [62]. It is interesting to note that the spin Josephson currents exist in the small Raman coupling region (such as $\Omega=0.001 E_{r}$ within the simulation time scale in Fig. 3), where the two spins are actually almost decoupled and then exhibit only independent external Josephson oscillations between the two wells. Therefore, the spin JEs referred to here actually come from the spin-dependent Josephson tunneling instead of spin flips. The spin Josephson currents in this $\Omega \rightarrow 0$ limit can be understood from the following picture of two special initial states. We consider a noninteracting pseudospin- $-\frac{1}{2}$ BEC in a double-well potential in the absence of SO coupling or any spin-dependent terms in the Hamiltonian. We consider a special initial state: All spin-up atoms are initially inside one of the wells, while all spin-down atoms are located in the other well. There are tunnelings between the two wells but without spin mixing. Under this initial condition, we can obtain a relation $I_{\uparrow}(t)=-I_{\downarrow}(t)$ because of the symmetry. So we have a nonzero spin oscillation $I_{s}=2 I_{\uparrow}(t)$, but the atomic current should vanish. On the other hand, if all spin-up and spin-down atoms are initially located in the same well (the other well is

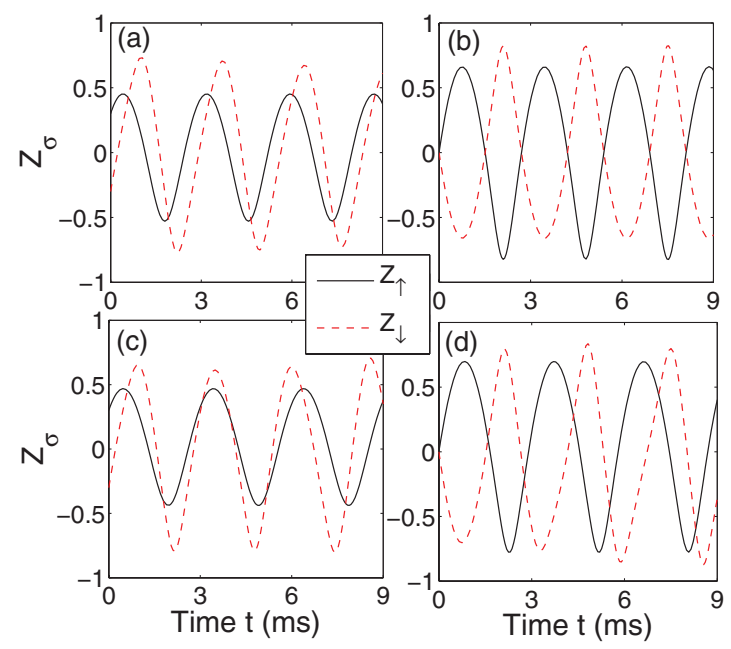

FIG. 3. (Color online) The time evolution of $\mathcal{Z}_{\sigma}$ for $\Omega=0.001 E_{r}$ in noninteracting limit. In (a) and (b) we have $\delta=0$ and $J_{\uparrow \uparrow}=$ $J_{\downarrow \downarrow}=-0.1 E_{r}$. In (c) and (d) we have $\delta=0.01 E_{r}, J_{\uparrow \uparrow}=-0.095 E_{r}$, and $J_{\downarrow \downarrow}=-0.105 E_{r}$. The initial conditions are $\mathcal{Z}_{\uparrow}(0)=-\mathcal{Z}_{\downarrow}(0)=$ $0.3, \phi_{\uparrow}(0)=0.5 \phi_{\downarrow}(0)=\pi / 8$ in (a) and (c) and $\mathcal{Z}_{\uparrow}(0)=\mathcal{Z}_{\downarrow}(0)=0$, $\phi_{\uparrow}(0)=-\phi_{\downarrow}(0)=\pi / 4$ in (b) and (d). 

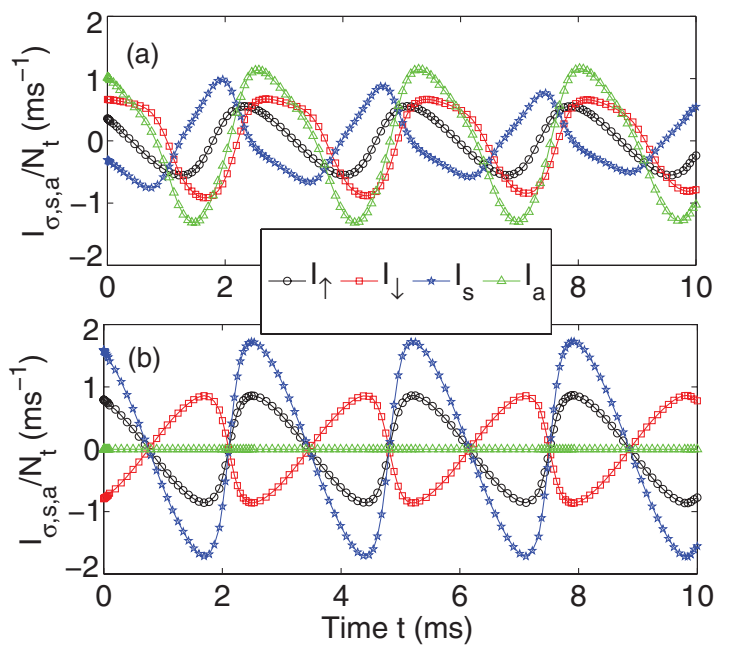

FIG. 4. (Color online) Josephson currents in the noninteracting limit. The time evolution of spin-dependent atomic currents $I_{\sigma}$, a net spin current $I_{s}$, and a total atomic current $I_{a}$ in (a) for $\mathcal{Z}_{\uparrow}(0)=-\mathcal{Z}_{\downarrow}(0)=0.3, \phi_{\uparrow}(0)=0.5 \phi_{\downarrow}(0)=\pi / 8$, and in (b) for $\mathcal{Z}_{\uparrow}(0)=\mathcal{Z}_{\downarrow}(0)=0, \phi_{\uparrow}(0)=-\phi_{\downarrow}(0)=\pi / 4$. Other parameters in (a) and (b) are $\delta=0, J_{\uparrow \uparrow}=J_{\downarrow \downarrow}=-0.1 E_{r}$ and $\Omega=0.001 E_{r}$.

totally empty), we have another relation $I_{\uparrow}(t)=I_{\downarrow}(t)$. Then the net spin current should vanish, while the atomic current $I_{a}=2 I_{\uparrow}(t)$ is nonzero. Under more general conditions, the external (spin or atomic) oscillations rely on the initial state (actually the phase and density differences of BECs between the two wells). However, the frequencies of both spin and atomic oscillations are independent of the initial states, as we see in the spectra calculated below.

For the weakly interacting case, we have to estimate the interaction energies $U_{\sigma \sigma}$ and $U_{\sigma \bar{\sigma}}$, which should be $U_{\sigma \sigma}, U_{\sigma \bar{\sigma}} \ll \hbar \omega$ due to the two-mode approximation. This requirement results in $U_{\sigma \sigma} \approx U_{\sigma \bar{\sigma}} \ll 0.1 E_{r}$. In this regime, the two spin Josephson tunneling processes are coupled via atomic interactions. To understand the effects of the interaction, we show in Fig. 5 some typical results of the time evolution of $\mathcal{Z}_{\sigma}$ for the same initial conditions and parameters in Fig. 3. It clearly demonstrates that the modification of $\mathcal{Z}_{\sigma}(t)$ due to atomic interactions is not significant and even very minor in some cases [such as the case for $\mathcal{Z}_{\sigma}(0)=0$ and $\delta=0$ in Figs. 3(b) and 5(b)] since the interaction energy is small compared with the tunneling energy. Therefore, the spin Josephson dynamics still exhibits a similar oscillatory feature in this regime.

To see more clearly the oscillatory properties of the spin JEs, we have numerically calculated the frequency spectra of the net spin currents $I_{s}$ for various conditions (such as those in Figs. 3 and 5). We find that the spectra for different cases exhibit a single peak centered at the slightly shifted frequency, as seen in Fig. 6. The single-peak feature shown in Fig. 6 implies that the spin current $I_{s}(t)$ can be described well by a sin function, while the weak interatomic interactions or the small Zeeman field can merely modify the period and amplitude of the current slightly. Thus we conclude that the spin JEs in this system are robust against the parameter adjustment and initial conditions.

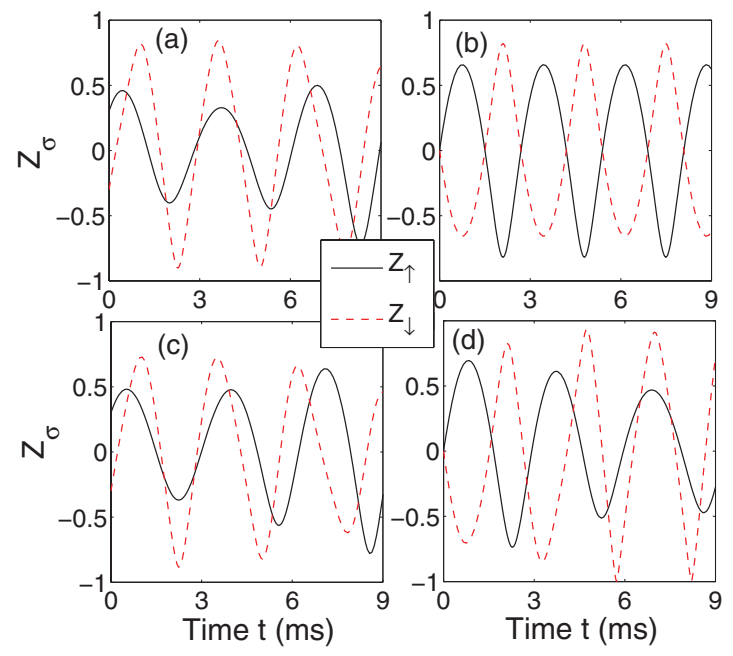

FIG. 5. (Color online) The time evolution of $\mathcal{Z}_{\sigma}$ in the weakly interacting regime with $U_{\sigma \sigma}=0.01 E_{r}$ and $U_{\sigma \sigma^{\prime}}=0.011 E_{r}$. Other parameters and initial conditions in (a)-(d) are the same with those in Figs. 3(a)-3(d), respectively.

\section{JOSEPHSON DYNAMICS IN OTHER RAMAN COUPLING REGIMES}

We now discuss briefly the Josephson dynamics of the system in other two regimes, the relatively strong and intermediate Raman coupling regimes. We first consider the relatively strong Raman coupling case (i.e., $\Omega \gg\left|J_{\sigma \sigma}\right|$ ), which can be realized such as by tuning $\Omega \lesssim 4 E_{r}$ (for $\left|J_{\sigma \sigma}\right| \sim 0.1 E_{r}$ or even smaller). In this regime, within the time scale $\hbar /\left|J_{\sigma \sigma}\right|$, one can approximately neglect the atomic interwell tunneling and just consider the internal dynamics in each single well, and thus the total particle number in well $j$ denoted by $N_{j t}=N_{j \uparrow}+N_{j \downarrow}$ is almost time-independent. Similarly, we define the normalized spin-imbalance in well $j$ as $\mathcal{Z}_{j}=\left[N_{j \downarrow}-N_{j \uparrow}\right] / N_{j t}$. The corresponding equations of motion for the noninteracting cases

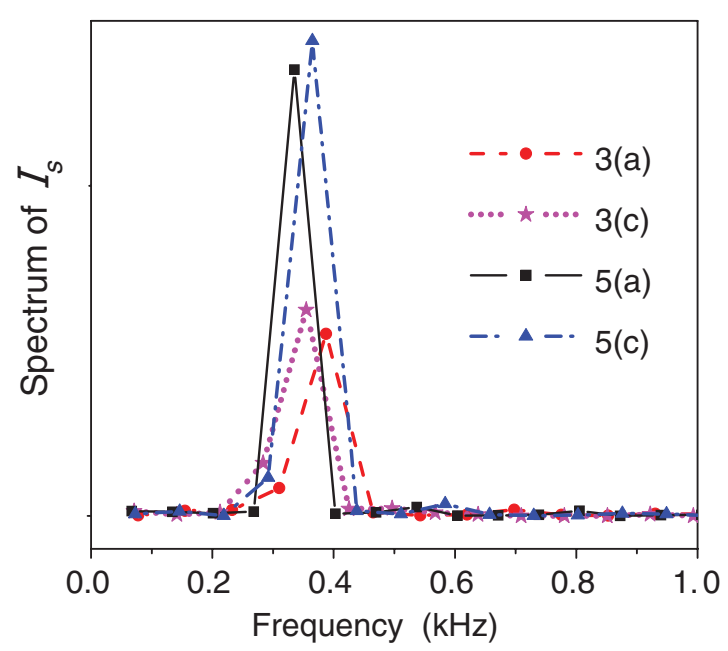

FIG. 6. (Color online) Spectra of the net spin currents $I_{s}$ for the cases in Figs. 3(a) and 3(c) and in Figs. 5(a) and 5(c). A single peak in the spectrum of each case implies that the spin current $I_{s}(t)$ is well described by a sin function. 
are then given by

$$
\begin{aligned}
& \dot{\mathcal{Z}}_{j}=-\frac{\Omega}{\hbar} \sqrt{1-\mathcal{Z}_{j}} \sin \phi_{j} \\
& \dot{\phi}_{j}=-\frac{\delta}{\hbar}+\frac{\Omega}{2 \hbar} \frac{\mathcal{Z}_{j}}{\sqrt{1-\mathcal{Z}_{j}^{2}}} \cos \phi_{j}
\end{aligned}
$$

Equations (27) describe the internal JEs [48] of the system. In this regime the Josephson oscillations are similar to those shown in the previous section. We note that the atomic tunneling between two spins (spin flipping) is induced by the Raman coupling, and thus such Josephson tunneling is in the spin space. In contrast to the regular BECs, the atomic gas with the synthetic SO coupling may condense with a finite but opposite momentum for different spins, and thus the internal JEs connect interesting macroscopic quantum tunneling in the momentum space, which has been observed in a more recent experiment of SO-coupled BECs in a harmonic trap [24].

In the intermediate regime, where $\Omega$ and $J_{\sigma \sigma}$ are comparable, we cannot obtain the Josephson equations in closed form similar to Eqs. (23) and (27) from the general equations of motion (19). Besides, there is an essential difference between weak (relatively strong) Raman coupling and intermediate coupling: $\mathcal{Z}_{\sigma}\left(\mathcal{Z}_{j}\right)$ is actually ill defined in the latter because $N_{L \sigma}+N_{R \sigma}\left(N_{j \uparrow}+N_{j \downarrow}\right)$ is varying, induced by spin flipping (interwell tunneling), with the same time scale as that of interwell tunneling (intrawell tunneling). So we consider $\rho_{\sigma}$ and $\rho_{j}$ in the intermediate Raman coupling region [see Eq. (20)], which also describe the spin-related external and the well-related internal JEs, respectively. For simplicity, we focus on the noninteracting case in this regime and present some typical numerical results based on Eq. (19).

Figure 7 shows the external Josephson oscillations $\rho_{\sigma}(t)$ and the internal Josephson oscillations $\rho_{j}(t)$ for both intermediate and small $\Omega$ cases. In Figs. 7(a) and 7(b), and Figs. 7(c) and $7(\mathrm{~d})$, we set $\Omega=0.1 E_{r}$ and $\Omega=0.25 E_{r}$, respectively; the oscillations of $\rho_{\sigma}(t)$ and $\rho_{j}(t)$ in these cases are no longer simple Rabi type as shown in Fig. 3 but exhibit complicated quasiperiod features [cf. Figs. 7(a)-7(d)]. The reason lies in the fact that the internal and external Josephson oscillations are coupled with the form described by Eq. (21), and their competitions are significant when $\Omega$ and $\left|J_{\sigma \sigma}\right|$ are comparable. In the very small $\Omega$ limit, the results of the spin-related external JEs in Fig. 7(e) return to those in Sec. IV, that is, almost decoupled Rabi oscillations; though the whole spin imbalance $\rho_{L}+\rho_{R}$ is nearly constant within the time scale of simulations, the spin imbalance in individual wells $\rho_{j}$ are still dynamic oscillations [cf. Fig. 7(f)], which arise from the external counterparts [see Eq. (21)]. In the opposite side, the well-related internal Josephson oscillations will recover as described by Eq. (27). Figures $7(\mathrm{~g})$ and $7(\mathrm{~h})$ show that the corresponding total external oscillation $\rho_{\uparrow}+\rho_{\downarrow}$. The total internal oscillation $\rho_{L}+\rho_{R}$ is decoupled, and each is of Rabi type, as seen from Eq. (21) (summing up $\rho_{\sigma}$ and $\rho_{j}$ makes the equation simple).

Finally, in this section, we examine the dynamics of the BECs, which initially localize in a momentum eigenstate of the single-particle SO Hamiltonian (2) [28]: the central momentum $k_{0}=k_{L} \sqrt{1-\left(\Omega / 4 E_{r}\right)^{2}}$ and the spinor
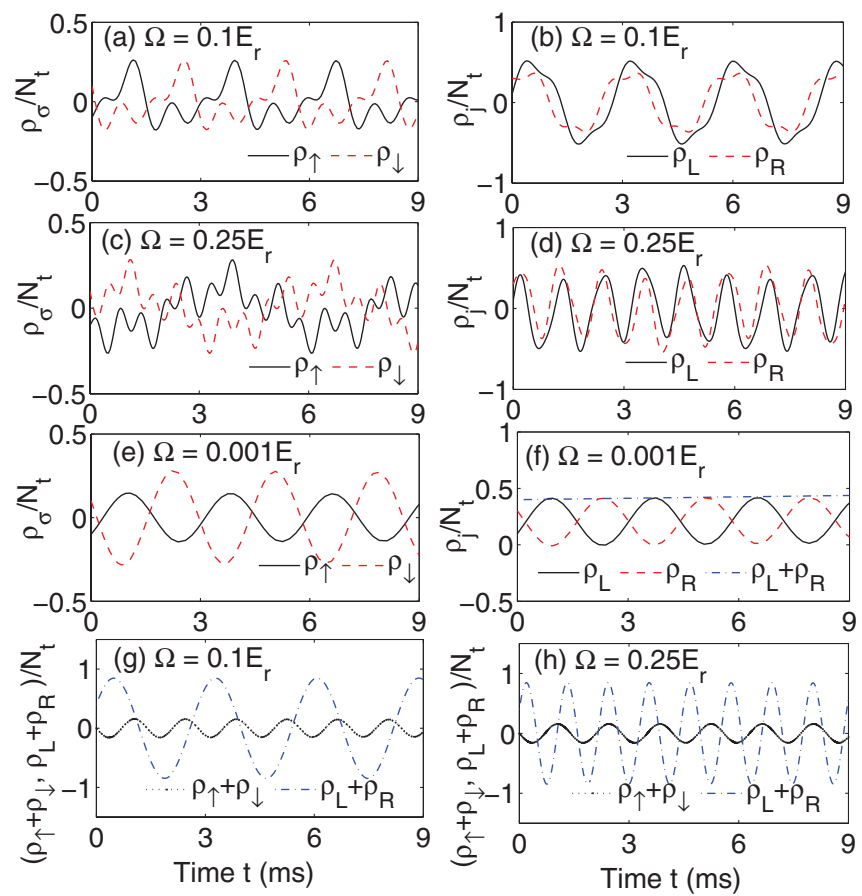

FIG. 7. (Color online) The time evolution of $\rho_{\sigma}$ and $\rho_{j}$ in the intermediate [(a)-(d)] and small [(e) and (f)] Raman coupling regimes. The corresponding time evolution of $\rho_{\uparrow}+\rho_{\downarrow}$ and $\rho_{L}+\rho_{R}$ are shown in $(\mathrm{g}),(\mathrm{h})$, and (f). The other parameters in (a)-(h) are $\delta=0$, $J_{\uparrow \uparrow}=J_{\downarrow \downarrow}=-0.1 E_{r}$, and $g_{\sigma \sigma^{\prime}}=0$. The initial conditions in (a)-(h) are $N_{L \uparrow}(0)=0.2 N_{t}, N_{L \downarrow}(0)=0.3 N_{t}, N_{R \uparrow}(0)=0.1 N_{t}, N_{R \downarrow}(0)=$ $0.4 N_{t}, \theta_{L \uparrow}(0)=-\theta_{L \downarrow}(0)=\pi / 8$, and $\theta_{R \uparrow}(0)=-\theta_{R \downarrow}(0)=\pi / 4$.

wave function $\left(\sin \frac{\xi}{2}, \cos \frac{\xi}{2}\right)^{T}$ with $\xi=\arcsin \frac{\Omega}{4 E_{r}}$. We further assume equal initial atomic number in the two wells, so that $N_{j \uparrow}(0)=0.5 N_{t}-N_{j \downarrow}(0)=0.5 \sin ^{2} \frac{\xi}{2} N_{t}$. The numerical results in Fig. 8 show similar characteristics of the external and internal Josephson oscillations as described above, without particular features for such initial eigenstates. We note that
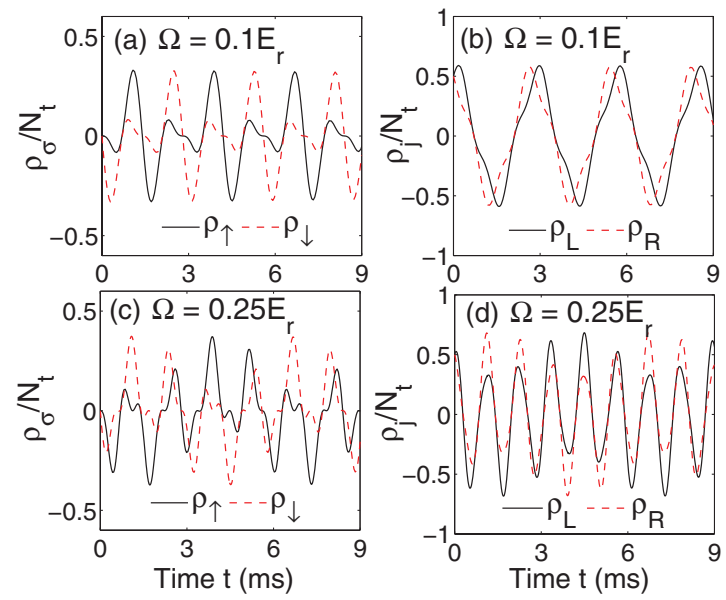

FIG. 8. (Color online) The time evolution of $\rho_{\sigma}$ and $\rho_{j}$ for a BEC initially localized in a momentum eigenstate of the single-particle SO Hamiltonian. The initial conditions in (a)-(d) are $N_{j \uparrow}(0)=0.5 N_{t}-N_{j \downarrow}(0)=0.5 \sin ^{2} \frac{\xi}{2} N_{t}$ with $\xi=\arcsin \frac{\Omega}{4 E_{r}}$, $\theta_{L \uparrow}(0)=-\theta_{L \downarrow}(0)=\pi / 8$, and $\theta_{R \uparrow}(0)=-\theta_{R \downarrow}(0)=\pi / 4$. The other parameters in (a)-(d) are $\delta=0, g_{\sigma \sigma^{\prime}}=0$, and $J_{\uparrow \uparrow}=J_{\downarrow \downarrow}=-0.1 E_{r}$. 
finite $\Omega$ shifts the central momentum from $\pm k_{L}$ [see Eq. (9)] to $\pm k_{0}$, and thus there is deviation in our estimation of the tunneling energies; however, the deviation is minor for $\Omega \lesssim E_{r}$ as the shift $\delta k=\left(k_{L}-k_{0}\right) / k_{L} \lesssim 3.2 \%$. Therefore, the basis given by Eq. (9) is still convenient for our discussions in the small and intermediate Raman coupling regimes.

\section{DISCUSSION AND CONCLUSION}

Before concluding, we make some additional comments related to possible concerns in realistic experiments: (i) For quasi-1D rubidium BECs, the typical interaction strength is $g_{\sigma \sigma^{\prime}} \sim 10^{-5} E_{r}$ for $l_{\perp} / l_{0} \sim 0.1\left(l_{0}\right.$ is about several micrometers) and $a_{\sigma \sigma^{\prime}} \sim 100 a_{0}$ with $a_{0}$ being the Bohr radius. Thus the two-mode approximation is applicable for $N_{t} \ll 10^{4}$, which is one order less than that of the realized SO-coupled BEC [20,24]. Therefore, one may prepare an initial SO-coupled $\mathrm{BEC}$ in a double-well trap with fewer atoms. To increase the atomic number within the two-mode regime, one can reduce the effective scattering length by Feshbach resonance [61]. However, a BEC with several thousands of atoms is substantially sufficient for the observation of the atomic JEs [50-53]. (ii) For a regular BEC without the synthetic SO coupling, the ratio between interaction energy $\left(\sim 0.01 E_{r}\right)$ and tunneling energy $\left(\left|J^{(T)}+J^{(V)}\right| \sim 0.001 E_{r}\right)$ can be about 10, and the BEC may exhibit MQST due to the nonlinear interactions [48,50]. For the SO-coupled BECs discussed in this paper, however, the tunneling energy is significantly increased and can be much larger than the interaction energy, so the presence of MQST in the external JEs is not anticipated in this regime (such as in Fig. 5). By decreasing $J^{(\mathrm{SO})}$, one can expect interesting spin-dependent MQST. (iii) The long-time behaviors of the external atomic tunneling for the small Raman coupling cases may be still affected by the spin flipping and may deviate from the oscillation feature previously described and need further considerations. (iv) At finite temperature, the thermal fluctuations and the depletion of BECs will renormalize the energy parameters such as $J_{\sigma \sigma^{\prime}}$ and $U_{\sigma \sigma^{\prime}}$ and thus generally damp the Josephson oscillations in experiments [50-53]. The detailed effects of damping and decoherence in this system under realistically experimental conditions require a separate treatment and further studies.

In summary, we have investigated the quantum dynamics of a SO-coupled BEC in a symmetric double-well potential. The SO coupling contributes to atomic tunneling between wells and significantly enhances JEs for realistic conditions. We have predicted a spin Josephson effect which can be observed in a practical experiment since all the required ingredients, including the SO-coupled BECs and the tunable double-well trapping potential, have already been achieved in the previous experiments.

\section{ACKNOWLEDGMENTS}

This work was supported in part by the NBRPC (No. 2011CBA00302), the SKPBRC (No. 2011CB922104 and No. 2011CB921503), the NSF of China (No. 11125417, No. 10974059, and No. 11075020), and the GRF (HKU7058/11P) and CRF (HKU8/CRF/11G) of the RGC of Hong Kong.
[1] M. V. Berry, Proc. Roy. Soc. London A 392, 45 (1984).

[2] C. P. Sun and M. L. Ge, Phys. Rev. D 41, 1349 (1990); S. L. Zhu and Z. D. Wang, Phys. Rev. Lett. 85, 1076 (2000); S. L. Zhu, Z. D. Wang, and Y. D. Zhang, Phys. Rev. B 61, 1142 (2000).

[3] F. Wilczek and A. Zee, Phys. Rev. Lett. 52, 2111 (1984); A. Zee, Phys. Rev. A 38, 1 (1988).

[4] For a review, see J. Dalibard, F. Gerbier, G. Juzeliünas, and P. Öhberg, Rev. Mod. Phys. 83, 1523 (2011); D. W. Zhang, Z. D. Wang, and S. L. Zhu, Front. Phys. 7, 31 (2012).

[5] R. Dum and M. Olshanii, Phys. Rev. Lett. 76, 1788 (1996).

[6] G. Juzeliūnas and P. Öhberg, Phys. Rev. Lett. 93, 033602 (2004).

[7] J. Ruseckas, G. Juzeliūnas, P. Öhberg, and M. Fleischhauer, Phys. Rev. Lett. 95, 010404 (2005).

[8] G. Juzeliūnas, J. Ruseckas, P. Öhberg, and M. Fleischhauer, Phys. Rev. A 73, 025602 (2006).

[9] S. L. Zhu, H. Fu, C. J. Wu, S. C. Zhang, and L. M. Duan, Phys. Rev. Lett. 97, 240401 (2006).

[10] X. J. Liu, X. Liu, L. C. Kwek, and C. H. Oh, Phys. Rev. Lett. 98, 026602 (2007).

[11] T. D. Stanescu, C. Zhang, and V. Galitski, Phys. Rev. Lett. 99, 110403 (2007)

[12] K. J. Günter, M. Cheneau, T. Yefsah, S. P. Rath, and J. Dalibard, Phys. Rev. A 79, 011604(R) (2009).

[13] G. Juzeliūnas, J. Ruseckas, and J. Dalibard, Phys. Rev. A 81, 053403 (2010).

[14] N. R. Cooper, Phys. Rev. Lett. 106, 175301 (2011).
[15] J. D. Sau, R. Sensarma, S. Powell, I. B. Spielman, and S. Das Sarma, Phys. Rev. B 83, 140510(R) (2011).

[16] D. L. Campbell1, G. Juzeliūnas, and I. B. Spielman, Phys. Rev. A 84, 025602 (2011).

[17] Z. F. Xu and L. You, e-print arXiv:1110.5705.

[18] S. L. Zhu, L.-B. Shao, Z. D. Wang, and L.-M. Duan, Phys. Rev. Lett. 106, 100404 (2011); L. Jiang, T. Kitagawa, J. Alicea, A. R. Akhmerov, D. Pekker, G. Refael, J. I. Cirac, E. Demler, M. D. Lukin, and P. Zoller, ibid. 106, 220402 (2011); S. L. Zhu, D. W. Zhang, and Z. D. Wang, ibid. 102, 210403 (2009).

[19] Y. J. Lin, R. L. Compton, A. R. Perry, W. D. Phillips, J. V. Porto, and I. B. Spielman, Phys. Rev. Lett. 102, 130401 (2009); Y. J. Lin, R. L. Compton, K. Jiménez-García, J. V. Porto, and I. B. Spielman, Nature (London) 462, 628 (2009); Y. J Lin, R. L. Compton, K. Jiménez-García, W. D. Phillips, J. V. Porto, and I. B. Spielman, Nat. Phys. 7, 531 (2011).

[20] Y.-J. Lin, K. Jiménez-García, and I. B. Spielman, Nature (London) 471, 83 (2011).

[21] Z. Fu, P. Wang, S. Chai, L. Huang, and J. Zhang, Phys. Rev. A 84, 043609 (2011).

[22] M. Aidelsburger, M. Atala, S. Nascimbëne, S. Trotzky, Y.-A. Chen, and I. Bloch, Phys. Rev. Lett. 107, 255301 (2011).

[23] J. Struck, C. Olschlager, R. Le Targat, P. Soltan-Panahi, A. Eckardt, M. Lewenstein, P. Windpassinger, and K. Sengstock, Science 333, 996 (2011). 
[24] S. Chen, J.-Y. Zhang, S.-C. Ji, Z. Chen, L. Zhang, Z.-D. Du, Y.-J. Deng, H. Zhai, and J.-W. Pan, e-print arXiv:1201.6018.

[25] T. D. Stanescu, B. Anderson, and V. Galitski, Phys. Rev. A 78, 023616 (2008).

[26] C. Wang, C. Gao, C. M. Jian, and H. Zhai, Phys. Rev. Lett. 105, 160403 (2010).

[27] C. M. Jian and H. Zhai, Phys. Rev. B 84, 060508 (2011).

[28] T. L. Ho and S. Zhang, Phys. Rev. Lett. 107, 150403 (2011).

[29] C. J. Wu, I. Mondragon-Shem, and X. F. Zhou, Chin. Phys. Lett. 28, 097102 (2011); X. F. Zhou, J. Zhou, and C. Wu, Phys. Rev. A 84, 063624 (2011).

[30] S. K. Yip, Phys. Rev. A 83, 043616 (2011).

[31] Z. F. Xu, R. Lü, and L. You, Phys. Rev. A 83, 053602 (2011).

[32] T. Kawakami, T. Mizushima, and K. Machida, Phys. Rev. A 84, 011607 (2011).

[33] E. van der Bijl and R. A. Duine, Phys. Rev. Lett. 107, 195302 (2011).

[34] S. Gopalakrishnan, A. Lamacraft, and P. M. Goldbart, Phys. Rev. A 84, 061604(R) (2011).

[35] S. Sinha, R. Nath, and L. Santos, Phys. Rev. Lett. 107, 270401 (2011).

[36] H. Hu, B. Ramachandhran, H. Pu, and X. J. Liu, Phys. Rev. Lett. 108, 010402 (2012).

[37] Q. Zhu, C. Zhang, and B. Wu, e-print arXiv:1109.5811.

[38] R. Barnett, S. Powell, T. Grass, M. Lewenstein, and S. Das Sarma, Phys. Rev. A 85, 023615 (2012).

[39] Y. Deng, J. Cheng, H. Jing, C.-P. Sun, and S. Yi, e-print arXiv:1110.0558.

[40] X.-Q. Xu and J. H. Han, Phys. Rev. Lett. 107, 200401 (2011).

[41] X. F. Zhou, J. Zhou, and C. J. Wu, Phys. Rev. A 84, 063624 (2011).

[42] J. Radić, T. A. Sedrakyan, I. B. Spielman, and V. Galitski, Phys. Rev. A 84, 063604 (2011).

[43] M. Merkl, A. Jacob, F. E. Zimmer, P. Öhberg, and L. Santos, Phys. Rev. Lett. 104, 073603 (2010).

[44] Y. Zhang, L. Mao, and C. Zhang, Phys. Rev. Lett. 108, 035302 (2012).
[45] D. W. Zhang, Z. Y. Xue, H. Yan, Z. D. Wang, and S. L. Zhu, Phys. Rev. A 85, 013628 (2012).

[46] J. Javanainen, Phys. Rev. Lett. 57, 3164 (1986).

[47] G. J. Milburn, J. Corney, E. M. Wright, and D. F. Walls, Phys. Rev. A 55, 4318 (1997).

[48] A. Smerzi, S. Fantoni, S. Giovanazzi, and S. R. Shenoy, Phys. Rev. Lett. 79, 4950 (1997); S. Raghavan, A. Smerzi, S. Fantoni, and S. R. Shenoy, Phys. Rev. A 59, 620 (1999).

[49] S. Giovanazzi, A. Smerzi, and S. Fantoni, Phys. Rev. Lett. 84, 4521 (2000).

[50] S. Levy, E. Lahoud, I. Shomroni, and J. Steinhauer, Nature (London) 449, 579 (2007).

[51] M. Albiez, R. Gati, J. Fölling, S. Hunsmann, M. Cristiani, and M. K. Oberthaler, Phys. Rev. Lett. 95, 010402 (2005).

[52] L. J. LeBlanc, A. B. Bardon, J. McKeever, M. H. T. Extavour, D. Jervis, J. H. Thywissen, F. Piazza, and A. Smerzi, Phys. Rev. Lett. 106, 025302 (2011).

[53] T. Betz, S. Manz, R. Bücker, T. Berrada, Ch. Koller, G. Kazakov, I. E. Mazets, H.-P. Stimming, A. Perrin, T. Schumm, and J. Schmiedmayer, Phys. Rev. Lett. 106, 020407 (2011).

[54] G. Thalhammer, G. Barontini, L. De Sarlo, J. Catani, F. Minardi, and M. Inguscio, Phys. Rev. Lett. 100, 210402 (2008).

[55] M.-S. Chang, Q. Qin, W. Zhang, L. You, and M. S. Chapman, Nat. Phys. 1, 111 (2005).

[56] X.-Q. Xu, L.-H. Lu, and Y.-Q. Li, Phys. Rev. A 78, 043609 (2008).

[57] B. Sun and M. S. Pindzola, Phys. Rev. A 80, 033616 (2009).

[58] H. Pu, W. P. Zhang, and P. Meystre, Phys. Rev. Lett. 89, 090401 (2002).

[59] S. Ashhab and C. Lobo, Phys. Rev. A 66, 013609 (2002).

[60] R. Qi, X.-L. Yu, Z. B. Li, and W. M. Liu, Phys. Rev. Lett. 102, 185301 (2009).

[61] C. Chin, R. Grimm, P. Julienne, and E. Tiesinga, Rev. Mod. Phys. 82, 1225 (2010).

[62] Y.-A. Chen, S. Nascimbène, M. Aidelsburger, M. Atala, S. Trotzky, and I. Bloch, Phys. Rev. Lett. 107, 210405 (2011). 